\title{
Indefinite Quasilinear Neumann Problem on Unbounded Domains
}

by

\section{J. CHABROWSKI}

Presented by Bogdan BOJARSKI

Summary. We investigate the solvability of the quasilinear Neumann problem (1.1) with sub- and supercritical exponents in an unbounded domain $\Omega$. Under some integrability conditions on the coefficients we establish embedding theorems of weighted Sobolev spaces into weighted Lebesgue spaces. This is used to obtain solutions through a global minimization of a variational functional.

1. Introduction. Let $\Omega \subset \mathbb{R}^{N}, N \geq 3$, be an unbounded domain with a smooth noncompact boundary $\partial \Omega$. We are mainly concerned with the nonlinear Neumann problem

$$
\left\{\begin{array}{cl}
-\operatorname{div}\left(\varrho(x)|\nabla u|^{p-2} \nabla u\right) & \\
=a(x)|u|^{q-2} u-b(x)|u|^{s-2} u-c(x)|u|^{t-2} u & \text { in } \Omega, \\
\varrho(x)|\nabla u|^{p-2} \frac{\partial}{\partial \nu} u(x)+h(x)|u|^{p-2} u=0 & \text { on } \partial \Omega,
\end{array}\right.
$$

where $\nu$ is the outward normal vector to $\partial \Omega$. The exponents $p, q, s$ and $t$ satisfy the conditions: $1<p<N, 1<s, q<p^{*}=N p /(N-p), p^{*}<t$. The coefficient $\varrho$ belongs to $L^{\infty}(\Omega) \cap L^{\infty}(\partial \Omega)$ and $0<\varrho_{\circ} \leq \varrho(x)$ a.e. for some constant $\varrho_{\circ}$. The coefficients $a$ and $b$ are allowed to change signs while $c$ is assumed to be nonnegative and measurable on $\Omega$. This problem was considered in the paper [9]. The authors of that paper established the existence of a nonnegative nontrivial solution assuming that $c \in L^{\infty}(\Omega)$,

2000 Mathematics Subject Classification: 35B33, 35J20, 35J65.

Key words and phrases: Neumann problem, sub- and supercritical exponents, indefinite weight functions. 
$b \geq 0$ and the coefficients $h, a$ and $b$ converge to 0 at a certain rate as $|x| \rightarrow \infty$. In this paper we consider this problem under different assumptions than those in [9]. More specifically, we assume that

$$
\begin{aligned}
& \int_{\Omega} \frac{|a|^{\frac{t}{t-q}}}{c^{\frac{q}{t-q}}} d x<\infty, \operatorname{Int}(\{x \in \Omega: a(x)>0\}) \neq \emptyset . \\
& \int_{\Omega} \frac{|b|^{\frac{t}{t-s}}}{c^{\frac{s}{t-s}}} d x<\infty .
\end{aligned}
$$

(H) There exist constants $0<c_{1}<c_{2}$ such that

$$
\frac{c_{1}}{(1+|x|)^{p-1}} \leq h(x) \leq \frac{c_{2}}{(1+|x|)^{p-1}}
$$

for a.e. $x \in \partial \Omega$.

Problems of the form (1.1) originate in applied sciences: nonlinear elasticity [6], mathematical biology [2] and also in differential geometry [8], [10]. Since the pioneering paper [4] problems of this nature have attracted considerable attention. We refer to two extensive survey articles [3] and [14] for a review of the current bibliography (see also [5] and [13]).

Solutions to problem (1.1) will be found through a variational approach. To describe the variational setting we define a suitable Sobolev space in the following way. By $C_{\delta}^{\infty}(\Omega)$ we denote the space $C_{0}^{\infty}\left(\mathbb{R}^{N}\right)$ restricted to $\Omega$. Let $w_{p}(x)=1 /(1+|x|)^{p}$ and let $E_{p}=E_{p}(\Omega)$ be the Sobolev space obtained as the completion of the space $C_{\delta}^{\infty}(\Omega)$ with respect to the norm

$$
\|u\|_{1, p}=\left(\int_{\Omega}|\nabla u|^{p} d x+\int_{\Omega}|u|^{p} w_{p}(x) d x\right)^{\frac{1}{p}} .
$$

By Lemma 1 in $[11]$ the norm $\|\cdot\|_{1, p}$ is equivalent to

$$
\|u\|_{E_{p}}=\left(\int_{\Omega}|\nabla u|^{p} \varrho(x) d x+\int_{\partial \Omega}|u|^{p} h(x) d S_{x}\right)^{\frac{1}{p}} .
$$

Given a nonnegative measurable function $w(x)$ on $\Omega$ we denote by $L^{r}(\Omega, w)$ the weighted Lebesgue space equipped with norm

$$
\|u\|_{L^{r}(\Omega, w)}=\left(\int_{\Omega}|u|^{r} w(x) d x\right)^{\frac{1}{r}} .
$$

We now define the underlying Sobolev space for problem (1.1) by $E(\Omega)=$ $E_{p}(\Omega) \cap L^{t}(\Omega, c)$ equipped with norm

$$
\|u\|_{E}=\|u\|_{E_{p}}+\left(\int_{\Omega}|u|^{t} c(x) d x\right)^{\frac{1}{t}} .
$$


Solutions to problem (1.1) will be obtained as critical points of the functional

$$
\begin{aligned}
J(u)= & \frac{1}{p}\left(\int_{\Omega}|\nabla u|^{p} \varrho(x) d x+\int_{\partial \Omega}|u|^{p} h(x) d S_{x}\right) \\
& -\frac{1}{q} \int_{\Omega}|u|^{q} a(x) d x+\frac{1}{s} \int_{\Omega}|u|^{s} b(x) d x+\frac{1}{t} \int_{\Omega}|u|^{t} c(x) d x .
\end{aligned}
$$

Throughout this paper, we denote strong convergence in a given Banach space $X$ by " $\rightarrow$ " and weak convergence by " $\rightarrow$ ". The norms in the Lebesgue spaces $L^{q}(\Omega)$ are denoted by $\|\cdot\|_{q}$.

The paper is organized as follows. In Section 2 we present a compact embedding theorem for the space $E(\Omega)$. This is used in Section 3 to establish the existence result for problem (1.1). In the proof of Theorem 1 in Section 3 we use some ideas from the paper [1].

2. Palais-Smale condition. First we establish the embedding of $E(\Omega)$ into a weighted Lebesgue space.

LEMMA 2.1. Let $w \geq 0$ be a function in $L_{\mathrm{loc}}^{\infty}(\Omega)$ such that

$$
\int_{\Omega} \frac{w^{\frac{t}{t-r}}}{c^{\frac{r}{t-r}}} d x<\infty
$$

where $1<r<p^{*}<t$. Then $E(\Omega)$ is compactly embedded into $L^{r}(\Omega, w)$.

Proof. Let $\delta>0$ and $R>0$. By the Young inequality we have

$$
\int_{\Omega}|u|^{r} w d x \leq \delta \int_{\Omega}|u|^{t} c d x+C_{1}(\delta) \int_{\Omega} \frac{w^{\frac{t}{t-r}}}{c^{\frac{r}{t-r}}} d x
$$

and

$$
\int_{\Omega_{R}}|u|^{r} w d x \leq \delta \int_{\Omega_{R}}|u|^{t} c d x+C_{1}(\delta) \int_{\Omega_{R}} \frac{w^{\frac{t}{t-r}}}{c^{\frac{r}{t-r}}} d x,
$$

where $\Omega_{R}=\Omega \cap\left(\mathbb{R}^{N}-B(0, R)\right)$ and $C_{1}(\delta)>0$ is a constant depending on $\delta, t$ and $r$. Let $\left\{u_{m}\right\}$ be a bounded sequence in $E(\Omega)$. We may assume that $u_{m} \rightarrow u$ in $L_{\text {loc }}^{r}(\Omega)$. Applying $(2.3)$ to $u_{m}-u$ we get

$$
\begin{aligned}
\int_{\Omega_{R}}\left|u_{m}-u\right|^{r} w d x & \leq \delta \int_{\Omega}\left|u_{m}-u\right|^{t} c d x+C_{1}(\delta) \int_{\Omega_{R}} \frac{w^{\frac{t}{t-r}}}{c^{\frac{r}{t-r}}} d x \\
& \leq \delta\left\|u_{m}-u\right\|_{E}^{t}+C_{1}(\delta) \int_{\Omega_{R}} \frac{w^{\frac{t}{t-r}}}{c^{\frac{r}{t-r}}} d x .
\end{aligned}
$$


By the Lebesgue dominated convergence theorem we have, for every $R>0$,

$$
\lim _{m \rightarrow \infty} \int_{\Omega \cap B(0, R)}\left|u_{m}-u\right|^{r} d x=0 .
$$

Since $\int_{\Omega_{R}}\left(w^{t /(t-r)} / c^{r /(t-r)}\right) d x \rightarrow 0$ as $R \rightarrow \infty$, the compactness of the embedding of $E(\Omega)$ into $L^{r}(\Omega, w)$ follows.

REMARK 2.2. It is known that $E_{p}(\Omega)$ is compactly embedded into $L^{r}\left(\Omega, w_{\alpha}\right)$, where $w_{\alpha}(x)=1 /(1+|x|)^{\alpha}$ and $(*) \alpha>N(1-r / t)$ (see [12]). Lemma 2.1 gives the compact embeddings of the subspace $E(\Omega)$ of $E_{p}(\Omega)$ into weighted Lebesgue spaces. If $c(x) \geq c_{\circ}>0$ on $\Omega$ for some constant $c_{\circ}$ (the function $c(x)$ can be unbounded on $\Omega$ ) and $\alpha$ satisfies $(*)$, then condition (2.1) holds with $w=w_{\alpha}$. Hence $E(\Omega)$ is compactly embedded into $L^{r}\left(\Omega, w_{\alpha}\right)$. We point out that we can deduce from Lemma 2.1 an embedding of $E(\Omega)$ into a weighted Lebesgue space with an unbounded weight function. For example, take $w(x)=(1+|x|)^{\alpha}$ and $c(x)=(1+|x|)^{\beta}$. If $\alpha, \beta>0$ and $N+\alpha t /(t-r)-\beta r /(t-r)<0$, then $E(\Omega)$ is compactly embedded into $L^{r}\left(\Omega,(1+|x|)^{\alpha}\right)$.

Lemma 2.3. Suppose (A), (B) and (H) hold. Then the functional $J$ is bounded from below.

Proof. It follows from (A), (B) and the Young inequality that

$$
\begin{aligned}
J(u) \geq & \frac{1}{p}\left(\int_{\Omega}|\nabla|^{p} \varrho d x+\int_{\partial \Omega}|u|^{p} h d S_{x}\right)+\left(\frac{1}{t}-2 \delta\right) \int_{\Omega}|u|^{t} c d x \\
& -C_{1}(\delta) \int_{\Omega} \frac{|a|^{\frac{t}{t-q}}}{c^{\frac{q}{t-q}}} d x-C_{2}(\delta) \int_{\Omega} \frac{|b|^{\frac{t}{t-s}}}{c^{\frac{s}{t-s}}} d x .
\end{aligned}
$$

Taking $2 \delta<1 / t$ yields the assertion.

We recall that a $C^{1}$-functional $\Phi: X \rightarrow \mathbb{R}$ on a Banach space $X$ satisfies the Palais-Smale condition at level $c\left((\mathrm{PS})_{c}\right.$ condition for short) if each sequence $\left\{x_{n}\right\} \subset X$ such that $(*) \Phi\left(x_{n}\right) \rightarrow c$ and $(* *) \Phi^{\prime}\left(x_{n}\right) \rightarrow 0$ in $X^{*}$ is relatively compact in $X$. Finally, any sequence $\left\{x_{n}\right\}$ satisfying $(*)$ and $(* *)$ is called a Palais-Smale sequence at level $c\left((\mathrm{PS})_{c}\right.$ sequence for short).

Lemma 2.4. The functional $J$ is of class $C^{1}$.

Proof. We write

$$
J(u)=\frac{1}{p}\left(\int_{\Omega}|\nabla u|^{2} d x+\int_{\partial \Omega}|u|^{p} h d S_{x}\right)-K_{a}(u)+K_{b}(u)+K_{c}(u),
$$

where

$$
K_{a}(u)=\frac{1}{q} \int_{\Omega}|u|^{q} a d x, \quad K_{b}(u)=\frac{1}{s} \int_{\Omega}|u|^{s} b d x, \quad K_{c}(u)=\frac{1}{t} \int_{\Omega}|u|^{t} c d x .
$$


Now we show that these functionals are of class $C^{1}$ on $E(\Omega)$. We only consider the functional $K_{b}$. The Gateaux derivative is given by

$$
\left\langle K_{b}^{\prime}(u), \phi\right\rangle=\int_{\Omega}|u|^{s-2} u \phi b d x
$$

for $\phi \in E(\Omega)$. Indeed, by the mean value theorem we have, for $0<t<1$,

$$
\left|\frac{b|u+t \phi|^{s}-b|u|^{s}}{|t|}\right| \leq s|b|(|u|+|\phi|)^{s-1}|\phi| .
$$

It follows from assumption (B) and the Hölder inquality that

$$
\begin{aligned}
\int_{\Omega}|b|(|u|+|\phi|)^{s-1}|\phi| d x \leq & \left(\int_{\Omega} \frac{|b|^{\frac{t}{t-s}}}{c^{\frac{s}{t-s}}} d x\right)^{\frac{t-s}{t}}\left(\int_{\Omega} c|u|^{\frac{s-1}{s}}|\phi|^{\frac{t}{s}} d x\right)^{\frac{s}{t}} \\
& +\left(\int_{\Omega} \frac{|b|^{\frac{t}{t-s}}}{c^{\frac{s}{t-s}}} d x\right)^{\frac{t-s}{t}}\left(\int_{\Omega}|\phi|^{t} c d x\right)^{\frac{s}{t}} \\
\leq & \left(\int_{\Omega} \frac{|b|^{\frac{t}{t-s}}}{\left.c^{\frac{s}{t-s}} d x\right)^{\frac{t-s}{t}}}\left(\int_{\Omega} c|u|^{t} d x\right)^{\frac{s-1}{t}}\left(\int_{\Omega}|\phi|^{t} c d x\right)^{\frac{1}{t}}\right. \\
& +\left(\int_{\Omega}^{\left.\frac{|b|^{\frac{t}{t-s}}}{c^{\frac{s}{t-s}}} d x\right)^{\frac{t-s}{t}}}\left(\int_{\Omega}|\phi|^{t} c d x\right)^{\frac{s}{t}} .\right.
\end{aligned}
$$

Since the right side of $(2.5)$ is in $L^{1}(\Omega)$ formula (2.4) follows from the Lebesgue dominated convergence theorem. To complete the proof it is enough to show that $K_{b}^{\prime}(u)$ is continuous on $E(\Omega)$. Let $u_{n} \rightarrow u$ in $E(\Omega)$. Since $u_{n} \rightarrow u$ in $L^{t}(\Omega, c)$ we may assume that, up to a subsequence, $c^{1 / t} u_{n} \rightarrow$ $c^{1 / t} u$ a.e. on $\Omega$ and that there exists a function $\zeta \in L^{t}(\Omega)$ such that $\left|c^{1 / t} u_{n}\right|,\left|c^{1 / t} u\right| \leq \zeta$ a.e. on $\Omega$. By the Hölder inequality we have, for $\phi \in E(\Omega)$,

$$
\begin{aligned}
\mid\left\langle K_{b}^{\prime}\left(u_{n}\right), \phi\right\rangle- & \left\langle K_{b}^{\prime}(u), \phi\right\rangle|=| \int_{\Omega}\left(\left|u_{n}\right|^{s-2} u_{n}-|u|^{s-2} u\right) \phi b d x \mid \\
\leq & \left(\left.\int_{\Omega}|| u_{n}\right|^{s-2} u_{n}-\left.|u|^{s-2} u\right|^{\frac{s}{s-1}}|b| d x\right)^{\frac{s-1}{s}}\left(\int_{\Omega}|\phi|^{s}|b| d x\right)^{\frac{1}{s}} \\
\leq & \left(\left.\int_{\Omega}|| u_{n}\right|^{s-2} u_{n}-\left.|u|^{s-2} u\right|^{\frac{s}{s-1} \frac{t}{s}} c d x\right)^{\frac{s-1}{t}}\left(\int_{\Omega} \frac{|b|^{\frac{t}{t-s}}}{c^{\frac{s}{t-s}}}\right)^{\frac{t-s}{t} \frac{s-1}{s}} \\
& \times\left(\int_{\Omega}|\phi|^{s}|b| d x\right)^{\frac{1}{s}} .
\end{aligned}
$$

By the Lebesgue dominated convergence theorem the right side of this inequality converges to 0 uniformly in $\phi$ on bounded subsets of $E(\Omega)$. 
Proposition 2.5. Suppose that assumptions (A), (B) and (H) hold. Assume additionally in the case $1<q, s<2$ that $a^{+} \in L_{\mathrm{loc}}^{\infty}(\Omega)$ and $b^{-} \in$ $L_{\mathrm{loc}}^{\infty}(\Omega)$. Then the functional $J$ satisfies the Palais-Smale condition.

Proof. Let $\left\{u_{n}\right\} \subset E(\Omega)$ be such that $J\left(u_{n}\right) \rightarrow c$ and $J^{\prime}\left(u_{n}\right) \rightarrow 0$ in $E(\Omega)^{*}$. Using the Young inequality we have, for large $n$,

$$
\begin{aligned}
\frac{1}{p}\left(\int_{\Omega}\left|\nabla u_{n}\right|^{p} \varrho d x+\int_{\partial \Omega}\left|u_{n}\right|^{p} h d S_{x}\right)+\frac{1}{t} \int_{\Omega}\left|u_{n}\right|^{t} c d x \\
\leq c+1+\frac{1}{q} \int_{\Omega}\left|u_{n}\right|^{q}|a| d x+\frac{1}{s} \int_{\Omega}\left|u_{n}\right|^{s}|b| d x \\
\leq c+1+\delta \int_{\Omega}\left|u_{n}\right|^{t} c d x+C(\delta)\left(\int_{\Omega} \frac{|a|^{\frac{t}{t-q}}}{c^{\frac{q}{t-q}}} d x+\int_{\Omega} \frac{|b|^{\frac{t}{t-s}}}{c^{\frac{s}{t-s}}} d x\right) .
\end{aligned}
$$

Taking $\delta<1 / t$ we deduce that $\left\{u_{n}\right\}$ is bounded in $E(\Omega)$. Hence we may assume that $u_{n} \rightarrow u$ in $E(\Omega)$. First, we consider the case $2<q$, s. Obviously in this case $t>2$. We set

$$
F(x, u)=a^{+}(x) \frac{|u|^{q}}{q}-\frac{c(x)}{4 t}|u|^{t}, \quad f(x, u)=F_{u}(x, u)
$$

and

$$
G(x, u)=b^{-}(x) \frac{|u|^{s}}{s}-\frac{c(x)}{4 t}|u|^{t}, \quad g(x, u)=G_{u}(x, u) .
$$

We now use the following inequality: for every $\alpha>0, \beta>0$ and $0<l<r$ we have

$$
\alpha|u|^{l}-\beta|u|^{r} \leq C_{l r} \alpha\left(\frac{\alpha}{\beta}\right)^{\frac{l}{r-l}}
$$

for every $u \in \mathbb{R}$, where the constant $C_{l r}>0$ depends only on $r$ and $l$. Applying this inequality we get

$$
\begin{aligned}
f_{u}(x, u) & =(q-1) a^{+}(x)|u|^{q-2}-(t-1) \frac{c(x)}{4}|u|^{t-2} \\
& \leq C_{t, q} a^{+}(x)\left(\frac{4 a^{+}(x)}{c(x)}\right)^{\frac{q-2}{t-q}}
\end{aligned}
$$

and

$$
\begin{aligned}
g_{u}(x, u) & =(s-1) b(x)^{-}|u|^{s-2}-(t-1) \frac{c(x)}{4}|u|^{t-2} \\
& \leq C_{s, t} b^{-}(x)\left(\frac{4 b^{-}(x)}{c(x)}\right)^{\frac{s-2}{t-s}} .
\end{aligned}
$$

Then it follows from (2.6) and (2.7) and the fact that $J^{\prime}\left(u_{n}\right) \rightarrow 0$ in $E(\Omega)^{*}$ 
that

$$
\begin{aligned}
& \int_{\Omega}\left(\left|\nabla u_{n}\right|^{p-2} \nabla u_{n}-\left|\nabla u_{m}\right|^{p-2} \nabla u_{m}\right)\left(\nabla u_{n}-\nabla u_{m}\right) \varrho d x \\
& +\int_{\partial \Omega}\left(\left|u_{n}\right|^{p-2} u_{n}-\left|u_{m}\right|^{p-2} u_{m}\right)\left(u_{n}-u_{m}\right) h d x \\
& +\int_{\Omega}\left(\left|u_{n}\right|^{q-2} u_{n}-\left|u_{m}\right|^{q-2} u_{m}\right)\left(u_{n}-u_{m}\right) a^{-} d x \\
& +\int_{\Omega}\left(\left|u_{n}\right|^{s-2} u_{n}-\left|u_{m}\right|^{s-2} u_{m}\right)\left(u_{n}-u_{m}\right) b^{+} d x \\
& +\frac{1}{2} \int_{\Omega}\left(\left|u_{n}\right|^{t-2} u_{n}-\left|u_{m}\right|^{t-2} u_{m}\right)\left(u_{n}-u_{m}\right) c d x \\
& =\int_{\Omega}\left(f\left(x, u_{n}\right)-f\left(x, u_{m}\right)\right)\left(u_{n}-u_{m}\right) d x \\
& +\int_{\Omega}\left(g\left(x, u_{n}\right)-g\left(x, u_{m}\right)\right)\left(u_{n}-u_{m}\right) d x+o(1) \\
& =\int_{\Omega}^{1} \int_{0}^{1} f_{u}\left(x, u_{n}+\sigma\left(u_{n}-u_{m}\right)\right) d \sigma\left(u_{n}-u_{m}\right)^{2} d x \\
& +\int_{\Omega}^{1} \int_{0}^{1} g_{u}\left(x, u_{n}+\sigma\left(u_{n}-u_{m}\right)\right) d \sigma\left(u_{n}-u_{m}\right)^{2} d x+o(1) \\
& \leq C_{t, q} \int_{\Omega} a^{+}\left(\frac{4 a^{+}}{c}\right)^{\frac{q-2}{t-q}}\left(u_{n}-u_{m}\right)^{2} d x+C_{s, t} \int_{\Omega} b^{-}\left(\frac{4 b^{-}}{c}\right)^{\frac{s-2}{t-s}} d x+o(1) .
\end{aligned}
$$

We may assume that $u_{n}-u_{m} \rightarrow 0$ in $L^{q / 2}\left(\Omega, a^{+}\right)$as $n, m \rightarrow \infty$. Since $\left(a^{+} / c\right)^{(q-2) /(t-q)} \in L^{q /(q-2)}\left(\Omega, a^{+}\right)$, we see that

$$
\lim _{n, m \rightarrow \infty} \int_{\Omega} a^{+}\left(\frac{a^{+}}{c}\right)^{\frac{q-2}{t-q}}\left(u_{n}-u_{m}\right)^{2} d x=0 .
$$

In a similar manner we show that

$$
\lim _{n, m \rightarrow \infty} \int_{\Omega} b^{-}\left(\frac{b^{-}}{c}\right)^{\frac{s-2}{t-s}}\left(u_{n}-u_{m}\right)^{2} d x=0 .
$$

To estimate from below the terms on the left side of (2.8) we use the following inequalities: for all $x, y \in \mathbb{R}^{N}$,

$$
\left(|x|^{r-2} x-|y|^{r-2} y, x-y\right) \geq C_{r}|x-y|^{r} \quad \text { if } r \geq 2,
$$


and for all $x, y \in \mathbb{R}^{N}$,

$$
C_{r} \frac{|x-y|^{2}}{(|x|+|y|)^{2-r}} \leq\left(|x|^{r-2} x-|y|^{r-2} y, x-y\right) \quad \text { if } r<2,
$$

where $C_{r}>0$ is a constant. If $p>2$ by $(2.9)$ we have

$$
C_{p} \int_{\Omega}\left|\nabla u_{n}-u_{m}\right|^{2} \varrho d x \leq \int_{\Omega}\left(\left|\nabla u_{n}\right|^{p} \nabla u_{m}-\left|\nabla u_{m}\right|^{p} \nabla u_{m}, \nabla u_{n}-\nabla u_{m}\right) d x .
$$

In this way we estimate the remaining terms of the left side of (2.8). If $1<p<2$, we use (2.10) to obtain

$$
\begin{aligned}
& \int_{\Omega}\left|\nabla u_{n}-\nabla u_{m}\right|^{p} \varrho d x \\
& \quad \leq \int_{\Omega} \frac{\left|\nabla u_{n}-\nabla u_{m}\right|^{p}}{\left(\left|\nabla u_{n}\right|-\left|\nabla u_{m}\right|\right)^{\frac{2-p}{2}} p}\left(\left|\nabla u_{n}\right|+\left|\nabla u_{m}\right|\right)^{\frac{2-p}{2} p} \varrho d x \\
& \quad \leq\left(\int_{\Omega} \frac{\left|\nabla u_{n}-\nabla u_{m}\right|^{2}}{\left(\left|\nabla u_{n}\right|+\left|\nabla u_{m}\right|\right)^{2-p}} \varrho d x\right)^{\frac{p}{2}}\left(\int_{\Omega}\left(\left|\nabla u_{n}\right|+\left|\nabla u_{m}\right|\right)^{p} \varrho d x\right)^{\frac{2-p}{2}} .
\end{aligned}
$$

Since the sequence $\left\{\int_{\Omega}\left|\nabla u_{n}\right|^{p} \varrho d x\right\}$ is bounded we derive from this that

$$
\begin{aligned}
& \left(\int_{\Omega}\left|\nabla u_{n}-u_{m}\right|^{p} \varrho d x\right)^{\frac{2}{p}} \\
& \quad \leq C_{1} \int_{\Omega}\left(\left|\nabla u_{n}\right|^{p-2} \nabla u_{n}-\left|\nabla u_{m}\right|^{p-2} \nabla u_{m}, \nabla u_{n}-\nabla_{m}\right) \varrho d x
\end{aligned}
$$

for some constant $C_{1}>0$. It is now clear that, up to a subsequence, $u_{n} \rightarrow u$ in $E(\Omega)$.

We now consider the case $1<q<2$. Let us denote by $I_{n m}$ the left hand side of inequality (2.8) without the integral involving $c$. We rewrite (2.8) in the following way:

$$
\begin{aligned}
I_{n m}+\int_{\Omega}\left(\left|u_{n}\right|^{t-2} u_{n}-\left|u_{m}\right|^{t-2} u_{m}, u_{n}-u_{m}\right) c d x \\
=\int_{\Omega}\left(\left|u_{n}\right|^{q-2} u_{n}-\left|u_{m}\right|^{q-2} u_{m}, u_{n}-u_{m}\right) a^{+} d x \\
\quad+\int_{\Omega}\left(\left|u_{n}\right|^{s-2} u_{n}-\left|u_{m}\right|^{s-2} u_{m}, u_{n}-u_{m}\right) b^{-} d x+o(1)
\end{aligned}
$$

if $1<s<2$. By Lemma 2.1 the last two integrals converge to 0 as $n, m \rightarrow \infty$. We now apply the argument from the previous case to the terms on the left side. In this way we again show that $u_{n} \rightarrow u$ in $E(\Omega)$. Finally, if $2<s$ we 
modify (2.8) in the following way:

$$
\begin{aligned}
I_{m n}+\frac{1}{2} \int_{\Omega}\left(\left|\nabla u_{n}\right|^{t-2} \nabla u_{n}-\left|\nabla u_{m}\right|^{t-2} \nabla u_{m}, \nabla u_{n}-\nabla u_{m}\right) c d x \\
=\int_{\Omega}\left(\left|u_{n}\right|^{q-2} u_{n}-\left|u_{m}\right|^{q-2} u_{m}, u_{n}-u_{m}\right) a^{+} d x \\
\quad+\int_{\Omega}^{1} \int_{0}^{1} \widetilde{f}_{u}\left(x, u_{n}+t\left(u_{n}-u_{m}\right)\right) d t\left(u_{n}-u_{m}\right)^{2} d x+o(1),
\end{aligned}
$$

where $\widetilde{f}_{u}=\widetilde{F}$ and $\widetilde{F}(x, u)=a^{+}(x)|u|^{q} / q-c(x)|u|^{t} / 2 t$. To complete the proof we repeat the argument from the previous part.

3. Main result. By Lemma 2.3 the functional $J$ is bounded from below on $E(\Omega)$. We put

$$
m=\inf _{u \in E(\Omega)} J(u)
$$

By the Ekeland variational principle [7] there exists a Palais-Smale sequence $\left\{u_{n}\right\}$ at level $m$ (see also [15, Corollary 2.5]). It then follows from Proposition 2.5 that, up to a subsequence, $u_{n} \rightarrow u$ in $E(\Omega)$. Obviously $u$ is a nontrivial solution of (1.1) provided $m<0$. In Theorem 1 below we formulate conditions guaranteeing that $m<0$. It is clear that $|u|$ is also a minimizer of $J$. Therefore we can assume that $u$ is nonnegative on $\mathbb{R}^{N}$. We put

$$
A(v)=\int_{\Omega}|v|^{q} a(x) d x \quad \text { and } \quad C(v)=\int_{\Omega}|v|^{t} c(x) d x .
$$

TheOREM 3.1. Suppose that (A), (B) and $(\mathrm{H})$ hold and moreover that $a^{+}, b^{-} \in L_{\mathrm{loc}}^{\infty}(\Omega)$.

(i) If (*) $q<\min (p, s, t)$, then problem (1.1) has a solution.

If $(*)$ is not satisfied we assume that $V=\operatorname{Int}\left(\operatorname{supp} a^{+}-\operatorname{supp} b\right) \neq \emptyset . W e$ then have two cases:

(ii) If $q<p$, then problem (1.1) has a nontrivial solution.

(iii) If $p<q$ and there exists a $C^{1}$ function $v$ with $\operatorname{supp} v \subset V$ such that

$$
(t-p) \frac{t^{\frac{p-q}{t-p}}}{p^{\frac{t-q}{t-p}}} \frac{(q-p)^{\frac{p-q}{t-p}}}{(t-q)^{\frac{t-q}{t-p}}} \frac{\left(\|v\|_{E_{p}}^{p}\right)^{\frac{t-q}{t-p}}}{C(v)^{\frac{p-q}{t-p}}}<A(v),
$$

then problem (1.1) has a nontrivial solution.

Proof. (i) Let $v$ be a $C^{1}$ function with $v \neq \equiv 0$ and $\operatorname{supp} v \subset\{x \in \Omega$ : $a(x)>0\}$. Since $q<\min (p, s, t)$ we see that $J(\sigma v)<0$ for $\sigma>0$ sufficiently small and so $m<0$. 
(ii) We choose $v$ as in (i) but with $\operatorname{supp} v \subset V$. Then $J(\sigma v)<0$ for $\sigma>0$ sufficiently small and so $m<0$.

(iii) Let $v$ be a function satisfying (3.1) and let

$$
f(\sigma)=\frac{\sigma^{p-q}}{p}\|v\|_{1, p}^{p}+\frac{\sigma^{t-q}}{t} C(v) .
$$

Then we have

$$
\inf _{\sigma>0} f(\sigma)=(t-p) \frac{t^{\frac{p-q}{t-p}}}{p^{\frac{t-q}{t-p}}} \frac{(q-p)^{\frac{p-q}{t-p}}}{(t-q)^{\frac{t-q}{t-p}}} \frac{\left(\|v\|_{E_{p}}^{p}\right)^{\frac{t-q}{t-p}}}{C(v)^{\frac{p-q}{t-p}}}<A(v) .
$$

Since $v$ satisfies (3.1) there exists $\sigma>0$ such that

$$
\frac{\sigma^{p-q}}{p}\|v\|_{E_{p}}^{p}+\frac{\sigma^{t-q}}{t} C(v)<A(v)
$$

and consequently $m<0$.

\section{References}

[1] S. Alama and G. Tarantello, Elliptic problems with nonlinearities indefinite in sign, J. Funct. Anal. 141 (1996), 159-215.

[2] D. G. Aronson and H. F. Weinberger, Multidimensional nonlinear diffusion arising in population genetics, Adv. Math. 30 (1978), 33-76.

[3] Th. Bartsch, Z. Q. Wang and M. Willem, The Dirichlet problem for superlinear elliptic equations, in: Handbook of Differential Equations, Vol. 2, M. Chipot and P. Quittner (eds.), Elsevier, 2005, 1-55.

[4] H. Brezis and L. Nirenberg, Positive solutions of nonlinear elliptic equations involving critical Sobolev exponents, Comm. Pure Appl. Math. 36 (1983), 437-477.

[5] J. Chabrowski and M. Willem, Least energy solutions of a critical Neumann problem with weight, Calc. Var. 15 (2002), 121-131.

[6] P. G. Ciarlet, Mathematical Elasticity, Vol. I, Three-Dimensional Elasticity, NorthHolland, Amsterdam, 1988.

[7] I. Ekeland, On the variational principle, J. Math. Anal. Appl. 47 (1974), 324-353.

[8] J. F. Escobar, Sharp constant in a Sobolev trace inequality, Indiana Univ. Math. J. 37 (1988), 687-698.

[9] D. A. Kandilakis and A. N. Lyberopoulos, Indefinite quasilinear elliptic problems with subcritical and supercritical nonlinearities on unbounded domains, J. Differential Equations 2006, 1-25, doi:10.1016/jde.2006.03.008.

[10] J. L. Kazdan, Prescribing the Curvature of a Riemannian Manifold, CBMS Reg. Conf. Ser. Math. 57, Amer. Math. Soc., Providence, RI, 1985.

[11] K. Pflüger, Existence and multiplicity of solutions to a p-Laplacian equation with nonlinear boundary condition, Electron. J. Differential Equations, 1998, no. 10, $13 \mathrm{pp}$.

[12] —, Compact traces in weighted Sobolev spaces, Analysis 18 (1998), 65-83.

[13] X. J. Wang, Neumann problems of semilinear elliptic equations involving critical Sobolev exponents, J. Differential Equations 93 (1991), 283-310. 
[14] J. D. Rossi, Elliptic problems with nonlinear boundary conditions and the Sobolev trace theorem, in: Handbook of Differential Equations, Vol. 2, M. Chipot and P. Quittner (eds.), Elsevier, 2005, 311-406.

[15] M. Willem, Minimax Theorems, Birkhäuser, Boston, 1996.

\section{J. Chabrowski \\ Department of Mathematics \\ University of Queensland \\ St. Lucia 4072, Qld, Australia \\ E-mail: jhc@maths.uq.edu.au}

Received September 7, 2006;

received in final form November 10, 2006 